\title{
Pathways to a diagnosis of autism spectrum disorder in Germany: a survey of parents
}

\author{
Juliana Höfer ${ }^{1 *}$, Falk Hoffmann ${ }^{1}$, Inge Kamp-Becker ${ }^{2}$, Luise Poustka ${ }^{3}$, Veit Roessner ${ }^{4}$, Sanna Stroth², Nicole Wolff ${ }^{4}$ \\ and Christian J. Bachmann ${ }^{5}$
}

\begin{abstract}
Background: Early identification of autism spectrum disorders (ASD) is a prerequisite for access to early interventions. Although parents often note developmental atypicalities during the first 2 years of life, many children with ASD are not diagnosed until school age. For parents, the long period between first parental concerns and diagnosis is often frustrating and accompanied by uncertainty and worry.

Methods: This study retrospectively explored the trajectories of children with a confirmed ASD diagnosis during the diagnostic process, from first parental concerns about their child's development until the definite diagnosis. A survey concerning the diagnostic process was distributed to parents or legal guardians of children with ASD from three specialized ASD outpatient clinics in Germany.

Results: The response rate was 36.9\%, and the final sample consisted of carers of 207 affected children (83.6\% male, mean age 12.9 years). The children had been diagnosed with childhood autism (55.6\%), Asperger syndrome (24.2\%), or atypical autism (20.3\%). On average, parents had first concerns when their child was 23.4 months old, and an ASD diagnosis was established at a mean age of 78.5 months. Children with atypical autism or Asperger syndrome were diagnosed significantly later (83.9 and 98.1 months, respectively) than children with childhood autism (68.1 months). Children with an $\mathrm{IQ}<85$ were diagnosed much earlier than those with an $\mathrm{IQ} \geq 85$. On average, parents visited 3.4 different health professionals ( $S D=2.4$, range 1-20, median: 3.0 ) until their child received a definite ASD diagnosis. Overall, $38.5 \%$ of carers were satisfied with the diagnostic process.
\end{abstract}

Conclusions: In this sample of children with ASD in Germany, the time to diagnosis was higher than in the majority of other comparable studies. These results flag the need for improved forms of service provision and delivery for suspected cases of ASD in Germany.

Keywords: Autism spectrum disorder, Autism, Diagnosis, Diagnostic process, Pathways, Satisfaction, Germany

\section{Introduction}

According to DSM-5, autism spectrum disorder (ASD) is characterized by persistent deficits in social communication and social interaction across multiple contexts accompanied by restricted, repetitive patterns of behavior, interests, or activities [1]. The prevalence of ASD has been steadily increasing in recent decades [2-4]. Its worldwide prevalence is now estimated at $1 \%[5,6]$, with

\footnotetext{
*Correspondence: juliana.hoefer@uni-oldenburg.de

1 Department of Health Services Research, Carl von Ossietzky University

Oldenburg, Ammerländer Heerstr. 140, 26129 Oldenburg, Germany

Full list of author information is available at the end of the article
}

the administrative prevalence in Germany being reported as $0.38 \%$ [2].

Besides environmental factors, genetics play a key role in the etiology of ASD, leading to abnormal brain development. The current treatment standard in children with ASD are early interventions, which focus on the improvement of social functioning, language and communication skills, and are effective in improving the long-term outcome in the above-mentioned domains. Accordingly, early identification of young children with ASD, and subsequent access to early interventions for these children is essential [5, 7-11]. In most Western countries a timely diagnosis of ASD also

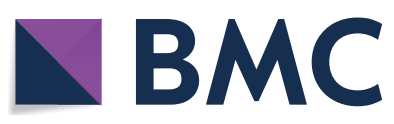

(c) The Author(s) 2019. This article is distributed under the terms of the Creative Commons Attribution 4.0 International License (http://creativecommons.org/licenses/by/4.0/), which permits unrestricted use, distribution, and reproduction in any medium, provided you give appropriate credit to the original author(s) and the source, provide a link to the Creative Commons license, and indicate if changes were made. The Creative Commons Public Domain Dedication waiver (http://creativecommons.org/ publicdomain/zero/1.0/) applies to the data made available in this article, unless otherwise stated. 
enables affected individuals and their families to get access to ASD specific health and social services.

Although in most cases ASD can be reliably diagnosed at the age of 24 months [12], with many parents reporting initial concerns even much earlier, a significant proportion of children are not diagnosed until school age $[11,13,14]$. For parents, this diagnostic delay is often accompanied by a long, frustrating period of uncertainty and worry, which can increase parental stress and dissatisfaction [15-17]. However, the symptomatology of older children and adolescents with ASD is often heterogeneous, and psychiatric comorbidities may complicate the differentiation of individuals with ASD from those with other diagnoses [18-21]. Moreover, ASD symptoms can appear in a variety of behavioural disorders, e.g. attention-deficit/ hyperactivity disorder, conduct disorder, or intellectual impairment [22]. Due to this symptom overlap, the diagnostic process of ASD can sometimes be difficult. For an accurate diagnosis of ASD, so-called "gold standard" psychometric instruments are employed, i.e. a standardized interview with the parents (Autism Diagnostic Interview-Revised, ADI-R), in combination with a semi-structured, standardized observation (Autism Diagnostic Observation Schedule, ADOS/ADOS-2) [4, 23-26]. Both special training and experience with these instruments are needed to apply them properly in order to reach a valid diagnosis [22,27].

In Germany, so far no standardised pathways for diagnostic assessment in cases of suspected ASD exist. Parents of children with suspected ASD are free to consult any health professional of their choice, without any need for referral. In general, in Germany paediatricians and general practitioners are the first point of contact for parents of children with mental health concerns [28].

A systematic review [13] shows that for ASD the mean age at diagnosis varies considerably between studies. The average age at ASD diagnosis ranged here from 38 to 120 months and has decreased over time. Besides clinical characteristics, the review revealed several other factors like sociodemographic characteristics or parental concern associated with age at diagnosis.

Although there exists already a considerable body of literature on the process of obtaining an ASD diagnosis, besides a large multi-country European study [29] that included data from Germany, there are only two studies exploring parents' experiences of the diagnostic process in Germany. One of these studies solely focussed on the time between age at first symptoms and age at diagnosis [30], while the other study included only children with Asperger syndrome [14]. Thus, for Germany, data related to professionals seen on the route to ASD diagnosis are not yet available.
Additionally, several studies have demonstrated an association between time to ASD diagnosis and the number of professionals consulted, respectively, and satisfaction of parents with the diagnostic process [15-17].

Against this background, the purpose of the study was to explore the experiences of parents of children and adolescents regarding the process of attaining an ASD diagnosis in Germany. Besides the time component of the diagnostic process, this survey also focused on the professionals consulted and the parental satisfaction with the diagnostic process.

\section{Methods}

This study is part of a large clinical and research network, the ASD-Net, focusing on the key challenges in ASD diagnostics, therapy and health service research [4].

\section{Recruitment and participants}

Between November 2015 and June 2016 three specialised child and adolescent psychiatric ASD outpatient clinics in Germany (Dresden, Mannheim and Marburg) contacted the parents/legal guardians of all children, who had a diagnosis of ASD and received services from or were diagnosed at the department. All patients were diagnosed using the ADOS/ADOS-2 and the ADI-R [4, $23,24]$. Inclusion criteria for the study were child's age under 19 years and a confirmed diagnosis of pervasive developmental disorder according to ICD-10.

\section{Questionnaire and instruments}

Parents of children with ASD completed a self-administered questionnaire on health-care services for ASD, based on the Client Service Receipt Inventory (CSRI) [31, 32], one of the most commonly used measures of health and social care service use. Together with a cover letter, a participant information sheet and a written informed consent form, the questionnaire has been usually mailed to parents. Only in exceptional cases, the survey documents were handed over personally. Depending on participating department, few weeks after the initial contact the parents received a reminder.

The participants had to give their consent, that their questionnaire data will be linked to data on, for instance, age, sex, clinical diagnosis (ICD 10-code), ADOS-2 comparison score and level of intellectual functioning of the child with ASD in a pseudonymised form. According to ICD-10, the level of intellectual functioning was divided into two groups: learning or intellectual disability (IQ $<85)$ vs. no learning or intellectual disability (IQ $\geq 85$ ). We further summarized the ADOS-2 comparison score into the following three different groups: minimal to low (score 1-4), moderate (score 5-7), and high (score 9-10) [33]. 
In addition to demographic characteristics the questionnaire asked parents about selected features of the diagnostic process. The items concerning the diagnostic process were based on previous studies on this topic [1517], and included (1) time taken to get an ASD diagnosis (age at first concerns, age at diagnosis), (2) professionals consulted because of first concerns, (3) outcome of first consultation, (4) professionals who made the final diagnosis, (5) number of professionals seen to get the ASD diagnosis and (6) satisfaction with the diagnostic process (using a 5-point Likert-scale, ranging from 'very dissatisfied' to 'very satisfied').

Children's age, the respondents' relationship to the child and the educational level of the parents, obtained from the questionnaire, were analysed for background information. To examine the highest parental educational level, participants were asked to provide information about mothers' and fathers' educational background. For analyses, the highest of the two levels was considered. The level of education was defined in accordance with the International Standard Classification of Education (ISCED) [34, 35] and has been classified into two groups: low/medium education (ISCED level 0-2) and high education (ISCED level 3). Referring to the German school system, low educational level complies with 9 years of schooling or leaving school without having acquired any school-leaving qualification. Medium educational level is equivalent to 10 years of schooling and high educational level complies with 12 or 13 years of schooling and a school-leaving qualification, which opens access to higher education institutions [36, 37].

Four parents of children with ASD, recruited from an outpatient department of child and adolescent psychiatry not participating in the study, pretested an initial version of the survey. On the basis of their comments, minor changes to the wordings of questions were made.

\section{Data analyses}

Baseline data were analysed using descriptive statistics. Mean ages with standard deviation (SD) concerning the diagnostic process were determined in the study population by age ( $0-11, \geq 12$ years), sex (male, female), ASD diagnosis (childhood autism, atypical autism, Asperger syndrome), intellectual functioning (no intellectual disabilities, intellectual disabilities), ADOS-2 comparison score (minimal till low, moderate, high) and highest parental educational level (low/medium, high). Regarding the classification of ASD diagnoses in this study, it should be noted that, unlike the DSM-5, the ICD-10 has not yet incorporated the concept of autism as a "spectrum disorder", and therefore offers different diagnostic categories for patients with autism.
Multivariable linear regression analyses were in each case performed to evaluate the associations between the above mentioned variables and the age at diagnosis as well as the age at first concerns. A logistic regression assessed the influence of several factors on parental satisfaction with the diagnostic process. Sensitivity analyses were performed using multiple imputation for missing values with 10 imputations. All statistical analyses were performed with SAS, version 9.4 (SAS Institute, Cary, USA).

The study protocol was reviewed and approved by the Commission for Impact Assessment Research and Ethics, Carl von Ossietzky University Oldenburg and by the ethic committees of the participating study sites.

\section{Results}

Baseline characteristics

637 survey documents were sent to parents/legal guardians of children with ASD, of which 49 documents could not be delivered due to a wrong address. 217 persons returned their questionnaire including a signed written consent (response: 36.9\%) and 211 questionnaires could be evaluated. Of these, four questionnaires had to be excluded because of implausible answers, resulting in a final sample of 207 respondents. $39.5 \%$ of the nonresponders and $34.3 \%$ of the responders were parents of children under 12 years. The baseline characteristics are presented in Table 1.

\section{Age at first concerns}

On average, parents reported that they first had concerns about their child's development when their child was 23.4 months old. $39.0 \%$ of parents noted developmental atypicalities during the 1st year of life, and one-fourth became concerned during 13 and 24 months (25.4\%). Parents of children in the older age group first suspected a difference in the development of their child later than parents of younger children (25.5 vs. 19.4 months) (Table 2). Parents of children with a diagnosis of childhood autism were first concerned at a mean age of 21.3 months whereas parents of children with Asperger syndrome were concerned later (29.8 months). Developmental abnormalities were noted earlier among children with an IQ $<85$ compared to children with an IQ $\geq 85$ (17.5 vs. 30.2 months).

Table 3 shows the results of the multivariable linear regression. Adjusting for all other covariates, parents of children in the older age group were concerned 8.4 months later than parents of younger children $(\mathrm{p}=0.0084)$ and parents of children with an IQ $<85$ noted atypicalities 13.0 months earlier than parents of children with an IQ $\geq 85(\mathrm{p}<0.0001)$. Sex, ASD diagnosis, ADOS-2 comparison score and the parental level of 
Table 1 Baseline characteristics

\begin{tabular}{|c|c|c|}
\hline Characteristics (number of respondents ${ }^{\mathrm{a}}$ ) & $\mathbf{n}$ & (\%) \\
\hline \multicolumn{3}{|l|}{$\operatorname{Sex}(n=207)$} \\
\hline Male & 173 & $(83.6)$ \\
\hline Female & 34 & $(16.4)$ \\
\hline Mean age, in years ( $\pm S D$; range) & 12.9 & $(3.4 ; 3-18)$ \\
\hline \multicolumn{3}{|l|}{ Age groups in years $(n=207)$} \\
\hline $0-11$ & 71 & $(34.3)$ \\
\hline$\geq 12$ & 136 & $(65.7)$ \\
\hline \multicolumn{3}{|l|}{ Diagnoses $(n=207)$} \\
\hline Childhood autism (F84.0) & 115 & $(55.6)$ \\
\hline Atypical autism (F84.1) & 42 & $(20.3)$ \\
\hline Asperger syndrome (F84.5) & 50 & $(24.2)$ \\
\hline \multicolumn{3}{|l|}{ Intellectual functioning $(\mathrm{n}=172)$} \\
\hline $\mathrm{IQ} \geq 85$ & 97 & $(56.4)$ \\
\hline $\mathrm{IQ}<85$ & 75 & $(43.6)$ \\
\hline \multicolumn{3}{|l|}{ ADOS-2 comparison score $(n=188)$} \\
\hline Minimal to low (1-4) & 27 & $(14.4)$ \\
\hline Moderate $(5-7)$ & 95 & $(50.5)$ \\
\hline High (8-10) & 66 & $(35.1)$ \\
\hline \multicolumn{3}{|l|}{ Highest parental level of education $(n=206)$} \\
\hline Low/middle & 80 & $(38.8)$ \\
\hline High & 126 & $(61.2)$ \\
\hline \multicolumn{3}{|l|}{ Respondents' relationship to the child $(n=206)$} \\
\hline Mother & 123 & $(59.7)$ \\
\hline Father & 18 & (8.7) \\
\hline Both parents & 57 & $(27.7)$ \\
\hline Grandparents or other relatives & 2 & $(1.0)$ \\
\hline Foster or adoptive parents & 6 & $(2.9)$ \\
\hline
\end{tabular}

${ }^{a}$ Due to missing values, figures may differ

education were not significantly associated with age at first concerns in multivariable analyses. Sensitivity analyses did not lead to any significant changes.

\section{Age at diagnosis}

In our sample, children received their ASD diagnosis at a mean age of 78.5 months (6.5 years) and $30.1 \%$ received the diagnosis during the first 48 months. Children in the younger group were diagnosed considerably earlier than children older than 11 years at the time of the survey (Table 2). Whereas children with childhood autism received their diagnoses at a mean age of 68.1 months (5.7 years), children with atypical autism and Asperger syndrome were diagnosed significantly later (at age 83.9 and 98.1 months, respectively). Diagnoses for children with an IQ $<85$ were made much earlier than for children with an IQ $\geq 85$. Children with a high ADOS-2 comparison score tended to be diagnosed earlier than children with a minimal till low score. Differences between the mentioned subgroups were statistically significant in the crude linear regression (Table 3).

Multivariable linear regression showed that children aged $>11$ years (at the time of the survey) were diagnosed 38.2 months later than younger children $(\mathrm{p}<0.0001)$. Compared to children with childhood autism, children with atypical autism received their diagnosis 19.7 months later, and those with Asperger syndrome were diagnosed 19.35 months later $(\mathrm{p}=0.0032, \mathrm{p}=0.0054)$. Children with an IQ $<85$ were diagnosed 15.5 months earlier than children with an $\mathrm{IQ} \geq 85(\mathrm{p}=0.0087)$. The remaining hypothesized predictors seen in Table 3 were not significantly associated with age at diagnosis in multivariable analyses, with sensitivity analyses yielding no significant changes.

The average time from first parental concerns and the establishment of a definite ASD diagnosis was 55.4 months (Table 2). Children aged $>11$ years at the time of the survey experienced a longer diagnostic delay than children in the younger group. The mean interval from concerns to definite diagnosis was longer for children with atypical autism (61.9 months), or Asperger syndrome (68.3 months), when compared to children with childhood autism (47.3 months).

\section{Referral trajectories}

Because of the first noted abnormalities with their child's development, the majority of parents first consulted a paediatrician (74.1\%). Only a minority of parents reported that they directly sought help at a social-paediatric centre (7.5\%), or a specialist for child and adolescent psychiatry (4.0\%). The outcome of the first consultation was mixed: $30.1 \%$ of parents were referred to another professional, and $27.6 \%$ were told that there was no problem with their child ("don't worry" or "he'll grow out of it"). In $17.2 \%$ of the cases a different diagnosis than ASD was given, and $11.3 \%$ were told to return if the problems persisted. The percentage of cases, where the final diagnosis was already made at the first consultation, was $7.4 \%$.

On average, parents visited 3.4 different professionals $(\mathrm{SD}=2.4$, range $1-20$, median: 3.0$)$ until their child received an ASD diagnosis. The diagnosis was most frequently made at a special outpatient clinic for ASD (48.5\%), or at an autism treatment centre (19.3\%).

\section{Satisfaction with the diagnostic process}

$38.5 \%$ of respondents were satisfied, $38.0 \%$ were dissatisfied and $23.4 \%$ were neither satisfied nor dissatisfied with the diagnostic process. Dissatisfaction was more frequent among parents of children with an IQ $<85$, compared to those with an IQ $\geq 85$. Prevalence of dissatisfaction was highest among parents of children with low ADOS-2 
Table 2 Mean age at first concerns and at diagnosis and mean time taken from first concerns to diagnosis stratified by characteristics

\begin{tabular}{|c|c|c|c|c|c|c|}
\hline \multirow[t]{2}{*}{ Characteristic } & \multicolumn{2}{|c|}{ Age at first concerns } & \multicolumn{2}{|c|}{ Age at diagnosis } & \multicolumn{2}{|c|}{$\begin{array}{l}\text { Time from first concerns } \\
\text { to diagnosis }\end{array}$} \\
\hline & $\mathrm{N}$ & Mean in months (SD) & $\mathrm{N}$ & Mean in months (SD) & $\mathrm{N}$ & Mean in months (SD) \\
\hline \multicolumn{7}{|l|}{ Sex } \\
\hline Male & 171 & $24.0(18.9)$ & 170 & $78.3(37.5)$ & 169 & $54.8(36.6)$ \\
\hline Female & 34 & $20.8(14.8)$ & 33 & $79.6(43.1)$ & 33 & $58.5(44.2)$ \\
\hline \multicolumn{7}{|l|}{ Age groups in years } \\
\hline $0-11$ & 69 & $19.4(15.1)$ & 70 & $55.7(21.6)$ & 69 & $36.5(24.5)$ \\
\hline$\geq 12$ & 136 & $25.5(19.5)$ & 133 & $90.5(39.9)$ & 133 & $65.2(39.9)$ \\
\hline \multicolumn{7}{|l|}{ ASD diagnosis } \\
\hline Childhood autism (F84.0) & 114 & $21.3(16.4)$ & 113 & $68.1(35.4)$ & 112 & $47.3(34.2)$ \\
\hline Atypical autism (F84.1) & 42 & $21.7(15.2)$ & 41 & $83.9(42.0)$ & 41 & $61.9(43.6)$ \\
\hline Asperger syndrome (F84.5) & 49 & $29.8(23.2)$ & 49 & $98.1(33.7)$ & 49 & $68.3(36.7)$ \\
\hline \multicolumn{7}{|l|}{ Intellectual functioning } \\
\hline $\mathrm{IQ} \geq 85$ & 95 & $30.2(21.2)$ & 94 & $89.3(36.6)$ & 93 & $59.9(37.1)$ \\
\hline $\mathrm{IQ}<85$ & 75 & $17.5(13.3)$ & 75 & $73.8(38.5)$ & 75 & $56.3(38.8)$ \\
\hline \multicolumn{7}{|l|}{ ADOS-2 comparison score } \\
\hline Minimal to low (1-4) & 27 & $28.1(18.7)$ & 25 & $94.4(42.5)$ & 25 & $67.4(38.3)$ \\
\hline Moderate (5-7) & 93 & $22.5(19.5)$ & 94 & $79.9(37.3)$ & 93 & $57.8(39.4)$ \\
\hline High $(8-10)$ & 66 & $22.0(15.8)$ & 65 & $70.9(37.5)$ & 65 & $48.8(34.5)$ \\
\hline \multicolumn{7}{|c|}{ Highest parental level of education } \\
\hline Low/middle & 80 & $25.4(18.1)$ & 79 & $82.1(41.0)$ & 79 & $56.9(41.7)$ \\
\hline High & 124 & $22.3(18.4)$ & 123 & $76.0(36.8)$ & 122 & $54.1(35.3)$ \\
\hline Overall & 205 & $23.4(18.3)$ & 203 & $78.5(38.4)$ & 202 & $55.4(37.8)$ \\
\hline
\end{tabular}

comparison scores, and decreased with increasing scores. Percentage of dissatisfaction increased with the number of professionals seen so get an ASD diagnosis (see Additional file 1).

The Additional file 1 shows the results of the logistic regression. Adjusting for all other covariates, the only factor significantly associated with dissatisfaction with the diagnostic process is the number of professionals seen during the process of obtaining a diagnosis. There were no significant changes in sensitivity analyses.

\section{Discussion}

In this study, children received their ASD diagnosis on average at an age of 6.5 years, with an interval of 4.6 years from first parental concerns to diagnosis. This finding is in line with a previous German study [30], that reported a mean age at ASD diagnosis of 6.3 years. Similar to our results (23.4 months), the Noterdaeme et al. study also found that the majority of parents became concerned of their child's development during the 2nd year of life. In the retrospective study of Kamp-Becker et al. [14], who analysed a cohort of children with Asperger syndrome in Germany, the mean age at diagnosis was much higher, i.e. 11.6 years. In $47.3 \%$ of cases, parents had first concerns regarding their child's development within the first 2 years of life, and in $43.2 \%$ concerns arose between age 3 and 6 . These results correspond to the observed differences between the ASD subgroups in this study. While in our study the mean age at diagnosis for children with Asperger syndrome was more than 3 years lower than in the study of Kamp-Becker et al. [14], children with Asperger syndrome were likewise diagnosed considerably later than children with other ASD diagnoses in our study. The lower age at diagnosis for children with Asperger syndrome in our study may also be a result of the more recent recruitment time period, in which both public and professional awareness of ASD, including Asperger syndrome, has increased.

In the multi-country European study of Salomone et al. [29] mean age at diagnosis was 3.5 years overall, and 4.0 years in Germany. The difference to our study, where the mean age at diagnosis was 6.5 years, can be explained by the different study designs: While the Salomone et al. study was a web-based survey, our study was clinic-based, thus having a bias towards more complex cases, which may be associated with higher age at diagnosis. Furthermore, Salomone et al. only recruited younger children (4 to 7 years of age), thus limiting age at diagnosis to a max. 
Table 3 Potential predictors for age at first concerns and age at diagnosis

\begin{tabular}{|c|c|c|c|c|}
\hline \multirow[t]{2}{*}{ Characteristic } & \multicolumn{2}{|c|}{ Age at first concerns in months ${ }^{a}$} & \multicolumn{2}{|c|}{ Age at diagnosis in months ${ }^{a}$} \\
\hline & $\begin{array}{l}\text { Crude linear regression } \\
\beta(95 \% \mathrm{CI})\end{array}$ & $\begin{array}{l}\text { Multivariable linear } \\
\text { regression }^{\mathrm{b}} \\
\beta(95 \% \mathrm{Cl})\end{array}$ & $\begin{array}{l}\text { Crude linear regression } \\
\beta(95 \% \mathrm{Cl})\end{array}$ & $\begin{array}{l}\text { Multivariable linear } \\
\text { regression }^{\mathrm{b}} \\
\beta(95 \% \mathrm{Cl})\end{array}$ \\
\hline \multicolumn{5}{|l|}{ Sex } \\
\hline Male & Reference & Reference & Reference & Reference \\
\hline Female & $-3.19(-9.90$ to 3.51$)$ & $-0.69(-8.38$ to 7.00$)$ & $1.28(-13.00$ to 15.56$)$ & $9.67(-4.33$ to 23.68$)$ \\
\hline \multicolumn{5}{|l|}{ Age groups in years } \\
\hline $0-11$ & Reference & Reference & Reference & Reference \\
\hline$\geq 12$ & 6.09 (0.86 to 11.31$)$ & 8.42 (2.16 to 14.68$)$ & 34.83 (24.84 to 44.83) & 38.23 (26.92 to 49.52) \\
\hline \multicolumn{5}{|l|}{ ASD diagnosis } \\
\hline Childhood autism (F84.0) & Reference & Reference & Reference & Reference \\
\hline Atypical autism (F84.1) & $0.37(-5.96$ to 6.71$)$ & $-2.05(-9.22$ to 5.12$)$ & $15.83(2.91$ to 28.76$)$ & 19.66 (6.58 to 32.73) \\
\hline Asperger syndrome (F84.5) & 8.41 (2.42 to 14.41$)$ & $2.52(-4.94$ to 9.97$)$ & 29.99 (17.86 to 42.12) & 19.35 (5.71 to 32.98) \\
\hline \multicolumn{5}{|l|}{ Intellectual functioning } \\
\hline $\mathrm{IQ} \geq 85$ & Reference & Reference & Reference & Reference \\
\hline $\mathrm{IQ}<85$ & $-12.72(-18.18$ to -7.27$)$ & $-12.96(-19.31$ to -6.62$)$ & $-15.51(-26.80$ to -4.21$)$ & $-15.53(-27.13$ to -3.93$)$ \\
\hline \multicolumn{5}{|l|}{ ADOS-2 comparison score } \\
\hline Minimal to low (1-4) & Reference & Reference & Reference & Reference \\
\hline Moderate (5-7) & $-5.61(-13.32$ to 2.10$)$ & $-2.40(-10.17$ to 5.36$)$ & $-14.51(-31.19$ to 2.17$)$ & $-5.91(-20.46$ to 8.63$)$ \\
\hline High (8-10) & $-6.14(-14.20$ to 1.92$)$ & $-3.27(-11.38$ to 4.84$)$ & $-23.44(-40.88$ to -5.99$)$ & $-11.43(-26.61$ to 3.76$)$ \\
\hline \multicolumn{5}{|c|}{ Highest parental level of education } \\
\hline Low/middle & Reference & Reference & Reference & Reference \\
\hline High & $-3.03(-8.14$ to 2.08$)$ & $-4.65(-10.41$ to 1.10$)$ & $-6.09(-16.90$ to 4.72$)$ & $-9.94(-20.50$ to 0.62$)$ \\
\hline
\end{tabular}

of 7 years, and probably also excluding most children with Asperger syndrome, who are often diagnosed significantly later. In contrast, our study had a broader age range, and our sample included a significant portion of patients with Asperger syndrome, resulting in higher age at diagnosis. Nevertheless, both in the Salomone et al. and in the current study, Germany is among those countries where age at diagnosis is highest.

In the review of Daniels and Mandell [13], who explored 42 studies from the period 1990-2012, in only three studies (one from France, one from the Czech Republic, and an older one from the UK) the average age at ASD diagnosis was higher than 6.5 years. In more recent studies, the age at ASD diagnosis was 4.6 years in a report from the UK [38], 3.9 years in a study from Norway [39], 4.4, 5.4 and 7.4 years, respectively, in three surveys from the USA [40, 41], and 6.2 years in a study from the Czech Republic [42].

\section{Time until diagnosis}

While in line with former studies from Germany, the comparably long time until diagnosis found in this study is nevertheless somewhat surprising, as Germany has a high-quality health system with generally good access to child and adolescent mental health services. There is a range of possible explanations for these findings, that encompass parental, professional, and health system factors. One possible reason is given in a study from the UK by Crane et al. [16], where parents usually waited a year from first concerns to initiating contact with a health professional. Waiting times for diagnostic appointments may also contribute to delayed diagnoses: In a Canadian study, the median waiting time for an ASD-specific diagnostic assessment was 7 months [43]. This also applies to Germany-as a result of the increased public awareness of ASD and the resulting requests for diagnostic ASD evaluations [4], the few existing specialised clinics for ASD in Germany are fully booked up, and long waiting lists are common. Unwarranted referrals also take up diagnostic capacities, as an US study by Monteiro et al. [44] demonstrated. In their study, a substantial portion of children without ASD were referred to scarce autism diagnostic resources, potentially delaying diagnosis and 
subsequent access to services for children who truly had an ASD [45].

The above-mentioned Canadian study [43] offers another reason for delays within the diagnostic process: The use of insufficient diagnostic tools, or of no diagnostic tools at all, may make subsequent consultations of further professionals necessary. The significant proportion of participants in our study with either Asperger syndrome (usually diagnosed much later than other forms of autism), or less pronounced ASD symptoms (more difficult to diagnose), may also have increased the average time to diagnosis. Additionally, all study participants came from specialized outpatient ASD clinics, which usually care for the most complex or difficult to diagnose patients. As this usually takes longer than in uncomplicated cases, this factor is another explanation for the longer time to diagnosis.

On a health system level, the prominent place of primary care physicians in the Germany health system [46] may also have contributed to the delay until diagnosis. In a UK study, nearly $40 \%$ of general practitioners had no substantial knowledge on ASD and the respective referral pathways [47]. In Germany, as mentioned above, no established referral pathways for children with suspected ASD exist, which may have contributed to both longer time to diagnosis, and to lower diagnostic quality [2]. The geographical settings of the participating centres may also have had an effect on age at ASD diagnosis [13], as in Germany the respective federal states differ significantly in terms of mental health resources [48].

\section{Factors associated with earlier diagnosis}

In our study, factors associated with an earlier age of diagnosis were childhood autism and learning disability or intellectual disability, respectively. While these factors largely comply with the German study from Noterdaeme et al. [30], as well as with the international literature [13], other well-known influencing factors like parental education were not significantly associated with earlier diagnosis in our study.

\section{Referral trajectories}

Regarding the number of professionals consulted until the final ASD diagnosis (3.4), comparable studies are scarce. Noticeably, the number of consulted professionals is closely related to the peculiarities of each national health system, and especially to its referral patterns. In an older study from the UK, Goin-Kochel et al. [15] reported an average of four to five (range 1-29) contacts to different clinicians on their way towards an ASD diagnosis, which is largely similar to our data. However, the fact that in our study on average it took 4.6 years from first parental concerns to diagnosis while parents consulted "only" 3.4 professionals during this period, is difficult to explain.

Nevertheless, the broad range of contacts with professionals in our study (up to 20) indicates a significant number of parents that have followed intertwined diagnostic pathways. In about half of patients in our sample, the definite diagnosis was made at a specialised ASD outpatient clinic. While this might reflect a recruitment bias, this proportion fits well with the figures of the web-based study of Goin-Kochel et al. [15], who reported the percentage of diagnoses at a specialist doctor at $46.8 \%$.

\section{Satisfaction with the diagnostic process}

The finding of nearly $40 \%$ dissatisfied parents is in line with the data from other surveys: In a study from the UK by Goin-Kochel et al. [15], 40.1\% of parents were not satisfied with the diagnostic process. Similarly, Jones et al. [49], who studied a sample of adults with ASD from the UK, found $40 \%$ of respondents to be very dissatisfied or quite dissatisfied. In a more recent study, Crane et al. [16] even found more than half of patients dissatisfied with the diagnostic process. A French survey by Chamak et al. [50] that evaluated parents' satisfaction with the way the ASD diagnosis was announced, yielded dissatisfaction rates of $63 \%$ (children), and 93\% (adults), respectively.

Regarding influencing factors, in our study only the number of professionals seen was associated with dissatisfaction with the diagnostic process, while age, level of education, or diagnostic subgroup were not. This is in contrast to other studies, who found a broader list of associated factors, including time to diagnosis, maternal education, manner of the diagnosing professional and family income $[15,16,51]$, and might be explained by the smaller sample size of our study.

\section{Strengths and limitations}

A strength of this study is the sample of children and adolescent with ASD diagnoses, which have been established using high-quality diagnostic standards [22]. An important limitation of this study is the low response rate, which limits generalisation of results. The relatively small sample size might have led to lower statistical power and large confidence intervals in multivariable analyses, and the wide range of patients' age in our sample caused large standard deviations for age at diagnosis and time to diagnosis. Due to the recruitment through specialized ASD outpatient clinics, our sample may be biased towards more complicated cases, which in turn might be associated with longer times to the definite ASD diagnosis. Because of the significant 
period between ASD diagnosis and this survey, there is also a risk for recall bias in parents' responses. For the sake of brevity, our questionnaire did not ask for e.g. ethnicity, comorbidity or family structure, which in some studies were factors associated with time to diagnosis $[13,52]$. Moreover, our study focused on the diagnostic process, thus missing out on the equally important post-diagnosis phase, e.g. provision of information on ASD-specific services [16], or time to receiving services [53]. The cross-sectional study design precluded an analysis of potential period effects, which have been reported in other studies [13, 38, 50], and may have affected the time to diagnosis in our study.

\section{Conclusions}

Concluding, our study shows that in the studied sample of children with ASD in Germany the time to diagnosis was higher than in the majority of international studies. This is regrettable, as a timely diagnosis is important in children with ASD, in order to enable them and their families to make use of social support and health services, and to improve their vocational outcome [54].

The results flag the need for better screening algorithms in primary care in Germany, especially for paediatricians, who were the first point of contact in three quarters of all cases in our study. Additionally, there is a need for clear referral criteria for children with suspected ASD, that allow a quicker and easier diagnostic process [55], in order to decrease time to diagnosis and increase parental satisfaction. The newly published German guidelines on diagnosis in suspected cases of ASD [26] will hopefully contribute to this aim. They recommend a two-step approach with first a diagnostic screening by healthcare professionals with experience in developmental disorders and second, in cases with substantiated suspicion, a referral to a specialised ASD outpatient clinic.

\section{Additional file}

Additional file 1. Prevalence of dissatisfaction of parents with the diagnostic process and factors associated with dissatisfaction.

\footnotetext{
Abbreviations

ADI-R: Autism Diagnostic Interview-Revised; ADOS: Autism Diagnostic Observation Schedule; ASD: autism spectrum disorder; Cl: confidence interval; CSRI: Client Service Receipt Inventory; DSM-5: Diagnostic and Statistical Manual of Mental Disorders, Fifth Edition; ICD-10: International Statistical Classification of Diseases and Related Health Problems, 10th Revision; ISCED: International Standard Classification of Education; SAS: Statistical Analysis Software; SD: standard deviation; UK: United Kingdom; USA: United States of America.
}

Authors' contributions

$\mathrm{JH}, \mathrm{FH}$ and CJB conceived of the study, participated in its design and coordination and drafted the manuscript; $\mathrm{JH}$ and $\mathrm{FH}$ performed the statistical analysis and interpretation of the data; IKB, LP, VR, SS and NW participated in the coordination of the study and performed the measurements. All authors read and approved the final manuscript.

\section{Author details \\ ${ }^{1}$ Department of Health Services Research, Carl von Ossietzky University Oldenburg, Ammerländer Heerstr. 140, 26129 Oldenburg, Germany. ${ }^{2}$ Depart- ment of Child and Adolescent Psychiatry, Psychosomatics and Psychotherapy, Philipps University Marburg, Hans-Sachs-Str. 4, 35039 Marburg, Germany. ${ }^{3}$ Department of Child and Adolescent Psychiatry, University Medical Center Göttingen, Von-Siebold-Str. 5, 37075 Göttingen, Germany. ${ }^{4}$ Department of Child and Adolescent Psychiatry, Medical Faculty of the Technical University Dresden, Fetscherstr. 74, 01307 Dresden, Germany. ${ }^{5}$ Department of Child and Adolescent Psychiatry, LVR-Klinikum Düsseldorf/Heinrich-Heine University Düsseldorf, Bergische Landstr. 2, 40629 Düsseldorf, Germany.}

\section{Acknowledgements}

The authors would like to thank Marie Kollarczyk, Imke Garten, Gerti Gerber, Friederike Helbig and Miriam-Sophie Petasch for their assistance in the conduct of this research and the respondents for participating in this study.

This article is dedicated to Prof. Dr. med. Dr. phil. Dr. h. c. Helmut Remschmidt, on the occasion of his 80th birthday, and in recognition of his clinical and scientific achievements for children and adolescents with autism spectrum disorders in Germany.

Competing interests

The authors declare that they have no competing interests.

\section{Availability of data and materials}

All data generated or analysed during this study are included in this published article.

Consent for publication

Not applicable.

\section{Ethics approval and consent to participate}

The study protocol was reviewed and approved by the Commission for Impact Assessment Research and Ethics, Carl von Ossietzky University Oldenburg (Reference Number DRs. 23/2015); and by the concerned institutional ethic committees (Heidelberg University: reference number 2015-607 N-MA; Marburg University: Reference Number 148/15; Dresden University: reference number EK6012016). The data protection concept was additionally coordinated with the Data Protection Officer of the Carl von Ossietzky University, Oldenburg.

\section{Funding}

This work was funded by the German Federal Ministry of Education and Research (FKZ 01EE1409F). The funding organisation had no influence on the design and conduct of the study, collection, management, analysis, and interpretation of the data, preparation, review, or approval of the manuscript, or the decision to submit the manuscript for publication.

\section{Publisher's Note}

Springer Nature remains neutral with regard to jurisdictional claims in published maps and institutional affiliations.

Received: 10 Auqust 2018 Accepted: 12 March 2019

Published online: 21 March 2019 


\section{References}

1. American Psychiatric Association. Diagnostic and statistical manual of mental disorders. 5th ed. Philadelphia: American Psychiatric Association 2013.

2. Bachmann CJ, Gerste B, Hoffmann F. Diagnoses of autism spectrum disorders in Germany: time trends in administrative prevalence and diagnostic stability. Autism. 2018;22(3):283-90.

3. Blumberg SJ, Bramlett MD, Kogan MD, Schieve LA, Jones JR, Lu MC. Changes in prevalence of parent-reported autism spectrum disorder in school-aged U.S. children: 2007 to 2011-2012. Natl Health Stat Report. 65. 2013:1-12.

4. Kamp-Becker I, Poustka L, Bachmann C, Ehrlich S, Hoffmann F, Kanske P, et al. Study protocol of the ASD-Net, the German research consortium for the study of Autism Spectrum Disorder across the lifespan: from a better etiological understanding, through valid diagnosis, to more effective health care. BMC Psychiatry. 2017;17(1):206.

5. Lai M-C, Lombardo MV, Baron-Cohen S. Autism. Lancet. 2014;383(9920):896-910.

6. Baron-Cohen S, Scott FJ, Allison C, Williams J, Bolton P, Matthews FE, et al. Prevalence of autism-spectrum conditions: UK school-based population study. Br J Psychiatry. 2009;194(6):500-9.

7. Swanson AR, Warren ZE, Stone WL, Vehorn AC, Dohrmann E, Humberd O. The diagnosis of autism in community pediatric settings: does advanced training facilitate practice change? Autism. 2014;18(5):555-61.

8. Rogers SJ, Vismara LA. Evidence-based comprehensive treatments for early autism. J Clin Child Adolesc Psychol. 2008;37(1):8-38.

9. Reichow B, Barton EE, Boyd BA, Hume K. Early intensive behavioral intervention (EIBI) for young children with autism spectrum disorders (ASD). Cochrane database Syst Rev. 2012;10(10):CD009260.

10. Warren Z, McPheeters ML, Sathe N, Foss-Feig JH, Glasser A, Veenstra-Vanderweele J. A systematic review of early intensive intervention for autism spectrum disorders. Pediatrics. 2011;127(5):e1303-11.

11. Zwaigenbaum L, Bauman ML, Stone WL, Yirmiya N, Estes A, Hansen RL, et al. Early identification of autism spectrum disorder: recommendations for practice and research. Pediatrics. 2015;136(136):S10-40.

12. Guthrie W, Swineford LB, Nottke C, Wetherby AM. Early diagnosis of autism spectrum disorder: stability and change in clinical diagnosis and symptom presentation. J Child Psychol Psychiatry Allied Discip. 2013;54(5):582-90.

13. Daniels AM, Mandell DS. Explaining differences in age at autism spectrum disorder diagnosis: a critical review. Autism. 2014;18(5):583-97.

14. Kamp-Becker I, Wulf C, Bachmann CJ, Ghahreman M, Heinzel-Gutenbrunner M, Gerber G, et al. Early symptoms of Asperger syndrome in childhood: a retrospective study. Kindheit und Entwicklung. Hogrefe Verlag Göttingen. 2010;19(3):168-76.

15. Goin-Kochel RP, Mackintosh VH, Myers BJ. How many doctors does it take to make an autism spectrum diagnosis? Autism. 2006;10(5):439-51.

16. Crane L, Chester JW, Goddard L, Henry LA, Hill E. Experiences of autism diagnosis: a survey of over 1000 parents in the United Kingdom. Autism. 2016;20(2):153-62.

17. Howlin P, Moore A. Diagnosis in autism: a survey of over 1200 patients in the UK. Autism. 1997;1(2):135-62.

18. Havdahl KA, Hus Bal V, Huerta M, Pickles A, Øyen A-S, Stoltenberg C, et al. Multidimensional influences on autism symptom measures: implications for use in etiological research. J Am Acad Child Adolesc Psychiatry. 2016;55(12):1054-1063.e3.

19. Hus V, Bishop S, Gotham K, Huerta M, Lord C. Factors influencing scores on the social responsiveness scale. J Child Psychol Psychiatry. 2013;54(2):216-24.

20. Kamp-Becker I, Langmann A, Stehr T, Custodis K, Poustka L, Becker K Zur Validität der deutschen Fassung der ADOS-2 unter Berücksichtigung von Geschlechtseffekten. Z Kinder Jugendpsychiatr Psychother. 2017;45(3):193-207

21. van Steensel FJA, Bögels SM, Wood JJ. Autism spectrum traits in children with anxiety disorders. J Autism Dev Disord. 2013;43(2):361-70.

22. Kamp-Becker I, Albertowski K, Becker J, Ghahreman M, Langmann A, Mingebach T, et al. Diagnostic accuracy of the ADOS and ADOS-2 in clinical practice. Eur Child Adolesc Psychiatry. 2018;27:1193-207.

23. Bölte S, Rühl D, Schmötzer G, Poustka F. ADI-R. Diagnostisches Interview für Autismus - Revidiert. Deutsche Fassung des Autism Diagnostic
Interview - Revised von Michael Rutter, Ann Le Couteur und Catherine Lord. 1. Auflage. Bern: Hans Huber; 2006

24. Rühl D, Bölte S, Feinein-Metthews S, Poustka F. ADOS, Diagnostische Beobachtungsskala für Autistische Störungen. 1st ed. Bern: Hans Huber; 2004

25. National Institute for Health and Care Excellence. Autism: recognition, referral and diagnosis of children and young people on the autism spectrum (NICE guideline). 2011. https://www.nice.org.uk/guidance/cg128. Accessed 05 Dec 2018.

26. DGKJP, DGPPN. Autismus-Spektrum-Störungen im Kindes-, Jugend- und Erwachsenenalter - Teil 1: Diagnostik. Interdisziplinäre S3-Leitlinie der DGKJP und der DGPPN sowie der beteiligten Fachgesellschaften, Berufsverbände und Patientenorganisationen. 2016. http://www.awmf.org/-leit inien/detail/II/028-018. Accessed 05 Dec 2018.

27. Elder J, Kreider C, Brasher S, Ansell M. Clinical impact of early diagnosis of autism on the prognosis and parent-child relationships. Psychol Res Behav Manag. 2017;10:283-92.

28. Wölfle S, Jost D, Oades R, Schlack R, Hölling H, Hebebrand J. Somatic and mental health service use of children and adolescents in Germany (KiGGS-study). Eur Child Adolesc Psychiatry. 2014:23:753-64.

29. Salomone E, Charman T, Mcconachie H, Warreyn P. Child's verbal ability and gender are associated with age at diagnosis in a sample of young children with ASD in Europe. Child Care Health Dev. 2016;42(1):141-5.

30. Noterdaeme M, Hutzelmeyer-Nickels A. Early symptoms and recognition of pervasive developmental disorders in Germany. Autism. 2010;14(6):575-88.

31. Beecham J, Knapp M. Costing psychiatric interventions. In: Thornicroft G, Brewin C, Wing J, editors. Measuring mental health needs. Measuring London: The Royal College of Psychiatrists; 1992. p. 163-83.

32. Personal Social Services Research Unit (PSSRU). What is the CSRI? | Client Service Receipt Inventory. https://www.pssru.ac.uk/csri/what-is-the-csri/. Accessed 05 Dec 2018

33. Lord C, Rutter M, DiLavore P, Risi S, Gotham K, Bishop S. Autism diagnostic observation schedule. 2nd ed. Los Angeles: Western Psychological Service; 2012.

34. UNESCO. International standard classification of education ISCED 1997. Paris: UNESCO; 1997.

35. UNESCO. International standard classification of education ISCED 2011. Paris: Montreal; 2012.

36. Schneider S. The international standard classification of education (ISCED-97) : an evaluation of content and criterion validity for 15 European countries. Mannheim: Mannheimer Zentrum fur Europaische Sozialforschung (MZES); 2008. p. 330.

37. Schroedter JH, Lechert Y, Lüttinger P. Die Umsetzung der Bildungsskala ISCED-1997 für die Volkszählung 1970, die Mikrozensus-Zusatzerhebung 1971 und die Mikrozensen 1976-2004 (Version 1). ZUMA-Methodenbericht, 2006/08. 2011:2006/08:IV,67.

38. Brett D, Warnell F, McConachie H, Parr JR. Factors affecting age at ASD diagnosis in UK: no evidence that diagnosis age has decreased between 2004 and 2014. J Autism Dev Disord. 2016;46(6):1974-84.

39. Larsen $\mathrm{K}$. The early diagnosis of preschool children with autism spectrum disorder in Norway: a study of diagnostic age and its associated factors. Scand J Child Adolesc Psychiatry Psychol. 2015;3(2):136-45.

40. Sheldrick RC, Maye MP, Carter AS. Age at first identification of autism spectrum disorder: an analysis of two US surveys. J Am Acad Child Adolesc Psychiatry. 2017;56(4):313-20.

41. Zuckerman K, Lindly OJ, Chavez AE. Timeliness of autism spectrum disorder diagnosis and use of services among U.S. elementary school-aged children. Psychiatr Serv. 2017;68(1):33-40.

42. Hrdlicka M, Vacova M, Oslejskova H, Gondzova V, Vadlejchova I, Kocourkova J, et al. Age at diagnosis of autism spectrum disorders: is there an association with socioeconomic status and family self-education about autism? Neuropsychiatr Dis Treat. 2016;12:1639-44.

43. Penner M, Anagnostou E, Ungar WJ. Practice patterns and determinants of wait time for autism spectrum disorder diagnosis in Canada. Mol Autism. 2018:9(1):16.

44. Monteiro SA, Spinks-Franklin A, Treadwell-Deering D, Berry L, SellersVinson S, Smith E, et al. Prevalence of Autism spectrum disorder in children referred for diagnostic autism evaluation. Clin Pediatr. 2015;54(14):1322-7. 
45. Murphy DGM, Beecham J, Craig M, Ecker C. Autism in adults. New biologicial findings and their translational implications to the cost of clinical services. Brain Res. 2011;1380:22-33.

46. Rattay P, Starker a, Domanska O, Butschalowsky H, Gutsche J, Kamtsiuris P. [Trends in the utilization of outpatient medical care in childhood and adolescence: results of the KiGGS study - a comparison of baseline and first follow up (KiGGS Wave 1)]. Bundesgesundheitsblatt Gesundheitsforschung Gesundheitsschutz. 2014

47. Unigwe S, Buckley C, Crane L, Kenny L, Remington A, Pellicano E. GPs' confidence in caring for their patients on the autism spectrum: an online self-report study. Br J Gen Pract. 2017;67(659):e445-52.

48. Bachmann CJ, Hoffmann F. Autismus-Spektrum-Störungen in Deutschland: Diagnoseprävalenz, medizinische Versorgung und zeitliche Trends. In: Klauber J, Gerste B, Robra B, Schmacke N, editors. VersorgungsReport 2015/2016 Schwerpunkt: Kinder und Jugendliche. Stuttgart: Schattauer; 2015. p. 167-84.

49. Jones L, Goddard L, Hill EL, Henry LA, Crane L. Experiences of receiving a diagnosis of autism spectrum disorder: a survey of adults in the United Kingdom. J Autism Dev Disord. 2014;44(12):3033-44.
50. Chamak B, Bonniau B, Oudaya L, Ehrenberg A. The autism diagnostic experiences of French parents. Autism. 2011;15(1):83-97.

51. Hidalgo NJ, McIntyre LL, McWhirter EH. Sociodemographic differences in parental satisfaction with an autism spectrum disorder diagnosis. J Intellect Dev Disabil. 2015;40(2):147-55.

52. Sicherman N, Loewenstein G, Tavassoli T, Buxbaum JD. Grandma knows best: family structure and age of diagnosis of autism spectrum disorder Autism. 2017;22:368-76.

53. Zablotsky B, Colpe LJ, Pringle BA, Kogan MD, Rice C, Blumberg SJ. Age of parental concern, diagnosis, and service initiation among children with autism spectrum disorder. Am J Intellect Dev Disabil. 2017;122(1):49-61.

54. Johnson TD, Joshi A. Dark clouds or silver linings? A stigma threat perspective on the implications of an autism diagnosis for workplace well-being. J Appl Psychol. 2016;101(3):430-49.

55. Osborne LA, Reed P. Parents' perceptions of communication with professionals during the diagnosis of autism. Autism. 2008;12(3):309-24.
Ready to submit your research? Choose BMC and benefit from:

- fast, convenient online submission

- thorough peer review by experienced researchers in your field

- rapid publication on acceptance

- support for research data, including large and complex data types

- gold Open Access which fosters wider collaboration and increased citations

- maximum visibility for your research: over 100M website views per year

At BMC, research is always in progress.

Learn more biomedcentral.com/submissions 